\title{
Considerations on the non-active power using geometric algebra
}

Article · May 2011

DOI: 10.1109/PowerEng.2011.6036438

CITATIONS

2

5 authors, including:

\section{Juan Carlos Bravo}

Universidad de Sevilla

27 PUBLICATIONS 109 CITATIONS

SEE PROFILE

Manuel Ordoñez

Universidad de Sevilla

28 PUBLICATIONS 101 CITATIONS

SEE PROFILE
READS

79

Juan-Carlos Montaño

Universidad de Sevilla

70 PUBLICATIONS 573 CITATIONS

SEE PROFILE

Some of the authors of this publication are also working on these related projects:

Project libera con la papaya View project 


\title{
Considerations on the Non-Active Power Using Geometric Algebra
}

\author{
M. Castilla, Member IEEE, J. C. Bravo, J. C. Montaño, Senior Member IEEE, \\ M. Ordóñez and A. López.
}

\begin{abstract}
Several approaches have been developed to define the non-active power concept under nonsinusoidal situations in electrical systems. Nevertheless, these contributions do not provide a complete and satisfactory solution to the non-active power reversibility between frequency domain and time domain. This paper presents a non-active power multivector concept, based on an original vector space frequency-domain approach that bridges the gap between both domains. The suggested correspondence can provide a convenient descriptive language to reconcile Fryze's instantaneous non-active power with Budeanu's deactive-power. To clarify this correspondence, a basis example is considered.
\end{abstract}

Index Terms-Non-active power, apparent power, instantaneous power, multivector, geometric algebra.

\section{INTRODUCTION}

\section{A. Motivation}

$\mathrm{O}$ ne of the most controversial issues in electrical engineering is the universal representation of the power equation of the electrical circuits in n-sinusoidal operation. Thus, while the concept of active power is fully accepted, the definition of power related to "reactive" and "harmonic" phenomena is still under discussion. This is of absolute importance, since it is the foundation of the non-active power concept that permits the design of suitable devices for the compensation of this power component. This paper gives a new vision on the representation of the non-active power $(\tilde{\Sigma})$ and suggests a multivectorial interpretation in a simple circuit with n-sinusoidal waveforms. From a theoretical standpoint, the approach presented here differs from the frequency domain approaches described in the literature by its emphasis on complex Geometric Algebra $\left(\mathcal{C G}_{n}\right)$. In this framework, the

This work was supported by the Ministry of Education and Science as part of a research through project DPI-2006-17467-CO2-01.

M. Castilla and J. C. Bravo are with the Electrical Engineering Department of the University of Sevilla, C/Virgen de África 7, (41011) - Sevilla, Spain. (phone:+34 9545528 47; fax: +34 9545516 88; e-mail: castilla@.us.es; carlos_bravo@us.es).

J. C. Montaño is with the Spanish Research Council (CSIC), Reina Mercedes Campus, POB 1052, 41080-Sevilla, Spain. (email: montano@irnas.csic.es).

M. Ordóñez is with the Department of Applied Mathematics II. Escuela, Politécnica Superior, Sevilla, Spain. (e-mail:mordonez@us.es).

A. López is with the Department of Electronic Technology, Escuela Politécnica Superior, Sevilla, Spain. (email: alojeda@us.es). non-active power is represented by a multivector that registers its magnitude, direction and sense. Moreover, in order to examine the reciprocity between frequency-domain and timedomain, there are some doubts which should be clarified. A rigorous treatment of this issue can lead to new avenues and perspectives in power theory.

\section{B. Literature review}

Budeanu and Fryze formulated the first power equations for n-sinusoidal operation between 1920 and 1930. The former, [1], addressed the question in the frequency domain, whilst the latter, [2], addressed the problem in the time domain.

Motivated by these theories, numerous valuable articles have appeared [3-13] although none considers the multivectorial character of apparent power components.

On the other hand, the multidimensionality of the power in $\mathrm{n}$-sinusoidal operation systems is the underlying obstacle that complicates the problem at hand. The power concept is better understood if a vector space is used for representation of the power equation. In this sense [14], [15] are two important and original contributions to the power analysis in linear/nonlinear operation. The large number of papers published on the physical and/or mathematical nature of these reversibility, suggests that work on this topic remains unfinished.

\section{Contributions}

The one-to-one correspondence between the non-active multivector terms and the instantaneous power components are considered and clarified. These objectives cannot be reached in a Complex Algebra framework.

\section{NON-ACTIVE POWER MULTIVECTOR}

Suppose that a nonsinusoidal voltage

$$
u(t)=\sqrt{2} \sum_{p \in N} U_{p} \sin \left(p \omega t+\alpha_{p}\right)
$$

is applied to a linear load, Fig.1, where $p$ is the harmonic order of $u(t)$. The resulting current has an instantaneous value given by

$$
i(t)=\sqrt{2} \sum_{q \in N} I_{q} \sin \left(q \omega t+\beta_{q}\right)
$$

where $q$ is the harmonic order of $i(t)$. For clarity of presentation and without loss of generality, the phases angles of the harmonic voltages are $\alpha_{p}=0$.

By applying the Clifford-Fourier transform [15] to the 
voltage (1) and current (2), the instantaneous quantities can be expressed as a linear combination of harmonic geometric phasors or complex-vectors in $\mathcal{C G}_{n}$

$$
\begin{gathered}
u(t)=\sqrt{2} \sum_{p} U_{p} \sin (p \omega t) \Rightarrow \tilde{U}=\sum_{p} U_{p} \sigma_{p} \\
i(t)=\sqrt{2} \sum_{q} I_{q} \sin \left(q \omega t+\varphi_{q}\right) \Rightarrow \tilde{I}=\sum_{q} I_{q} e^{j \varphi_{q}} \sigma_{q}
\end{gathered}
$$

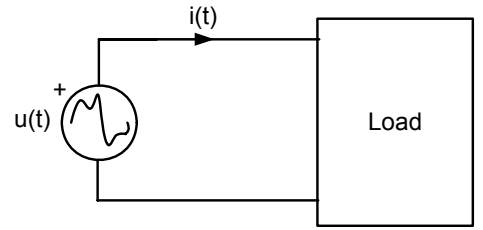

Fig. 1. Linear load with $\mathrm{n}$-sinusoidal waveforms

In this $\mathcal{C G}_{n}$ framework, the power multivector $\tilde{S}$ [15] entering the one-port in Fig. 1, is

$$
\tilde{S}=\tilde{U} \odot \tilde{I}^{*}=\{\underbrace{\tilde{U} \cdot \tilde{I}^{*}}_{\tilde{\Omega}^{*}} \oplus \underbrace{\tilde{U} \wedge \tilde{I}^{*}}_{\tilde{\Omega}^{\wedge}}\}
$$

where $\quad \tilde{U}=\sum_{p \in N}\left\|\tilde{U}_{p}\right\| \sigma_{p} \quad$ and $\quad \tilde{I}^{*}=\sum_{q \in N}\left\|I_{q}\right\| e^{j \varphi_{q}} \sigma_{q}$ are the geometric phasors of voltage and conjugate current respectively. In the expanded form of (5), the two components $\tilde{\Omega}^{\bullet}$ (scalar complex) and $\tilde{\Omega}^{\wedge}$ (bivector complex) can be expressed

$$
\begin{gathered}
\tilde{\Omega}^{\bullet}=\operatorname{Re}\left\|\left\{\tilde{\Omega}^{\bullet}\right\}\right\|+j \operatorname{Im}\left\|\left\{\tilde{\Omega}^{\bullet}\right\}\right\|=\sum_{P}\left(U_{p} I_{p} \cos \varphi_{p}+j U_{p} I_{p} \sin \varphi_{p}\right) \sigma_{0} \\
=\sum_{P}\left(P_{p}+j Q_{p}\right) \sigma_{0}=(P+j Q) \sigma_{0} \\
\tilde{\Omega}^{\wedge}=\sum_{p \neq q} U_{p} I_{q} e^{j \varphi_{q}} \sigma_{p q}=\sum_{p \neq q}\left(\Delta_{p q}+j \Lambda_{p q}\right) \sigma_{p q}
\end{gathered}
$$

where $\left\|\operatorname{Re}\left\{\tilde{\Omega}^{\bullet}\right\}\right\|$ is the active power $\mathrm{P}$ and $\left\|\operatorname{Im}\left\{\tilde{\Omega}^{\bullet}\right\}\right\|$ is associated to Budeanu and Slonim's reactive power $Q$. Combining (6) and (7), we obtain the non-active power multivector $\tilde{\Sigma}$ that is defined as

$$
\tilde{\Sigma}=\operatorname{Im}\left[\tilde{\Omega}^{\cdot}\right]+\sum_{p \neq q}\left(\Delta_{p q}+j \Lambda_{p q}\right) \sigma_{p q}
$$

where the terms $\left(\Delta_{p q}+j \Lambda_{p q}\right)$ represents the interaction of voltage and current harmonics with different frequencies. The suggested representation (8) can be extended in a straightforward manner to the existing power equations in the frequency domain. In this way, the $\tilde{\Sigma}$ multivector enables separate treatment of the smallest non-active power term which considers only the fundamental component, Emanuel approach [11], and the largest one which includes the imaginary part of the complex scalar and the total complex bivector, Fryze approach [2]. It is remarkable that the quantities proposed in (5) have three basic attributes: magnitude, direction and sense.

\section{POWERS IN FREQUENCY-DOMAIN AND TIME -DOMAIN}

The inner or scalar product in (5) is symmetric but, on the other hand, the outer product is antisymmetric $\left(\sigma_{p q}=-\sigma_{q p}\right)$. Thus the apparent power (5) and its norm can be expressed as

$$
\begin{aligned}
& \tilde{S}=\sum_{P}\left(P_{p}+j Q_{p}\right) \sigma_{0}+\sum_{p \neq q}\left(\left(\Delta_{p q}-\Delta_{q p}\right)+j\left(\Lambda_{p q}-\Lambda_{q p}\right)\right) \sigma_{p q} \\
& \|\tilde{S}\|^{2}=P^{2}+Q^{2}+\left[\sum_{p \neq q}\left(\Delta_{p q}-\Delta_{q p}\right)\right]^{2}+\left[\sum_{p \neq q}\left(\Lambda_{p q}-\Lambda_{q p}\right)\right]^{2}
\end{aligned}
$$

and the non-active power multivector is now

$$
\tilde{\Sigma}=\underbrace{j \sum_{P} Q_{p} \sigma_{0}}_{\text {Scalar part }}+\underbrace{\sum_{p \neq q}\left(\left(\Delta_{p q}-\Delta_{q p}\right)+j\left(\Lambda_{p q}-\Lambda_{q p}\right)\right)}_{\text {Bivector part }} \sigma_{p q}
$$

which are expressed as a linear combination of the multivector basis.

In time domain, the instantaneous power $s(t)$ entering the generic circuit of Fig. 1 is given by

$$
s(t)=u(t) i(t)
$$

Now it is seen from (3),(4) and (12) that it may be written in the form

$$
\begin{aligned}
& s(t)=\sum_{\mathrm{p}} \underbrace{\overbrace{U_{p} I_{p} \cos \varphi_{p}}^{P_{p}}\left[2 \sin ^{2} p \omega t\right]}_{p_{p}(t)} \\
& \underbrace{-\overbrace{\left(U_{p} I_{p} \sin \varphi_{p}\right)}^{Q_{p}}[2 \sin (p \omega t) \cos (p \omega t)]}_{q_{p}(t)} \\
& +\underbrace{\overbrace{U_{p} I_{q} \cos \varphi_{q}}^{\Delta_{p q}}[2 \sin (p \omega t) \sin (q \omega t)]}_{p_{p q}(t)} \\
& \underbrace{-\overbrace{\left(U_{p} I_{q} \sin \varphi_{q}\right)}^{\Lambda_{p q}}[2 \sin (p \omega t) \cos (q \omega t)]}_{q_{p q}(t)}
\end{aligned}
$$

where $p_{p}(t)$ is the instantaneous harmonic active power term, $q_{p}(t)$ is the instantaneous harmonic reactive power term, and $p_{p q}(t)$ and $q_{p q}(t)$ are the instantaneous crossharmonic power terms. This analysis can readily be extended to more general situations involving any number of harmonic components, as long as the suitable $\mathcal{C G}_{n}$ structure is chosen.

Therefore, (13) consist of two parts, where the first part contains a group of instantaneous power terms involving likefrequency and the second part contains those involving crossfrequency. Then, the total instantaneous power $s(t),(13)$, can be seen as formed by two power components, active $p_{a}(t)$, and non active $p_{n a}(t)$, given by

$$
\begin{gathered}
p_{a}(t)=\sum_{p} p_{p}(t) \\
p_{n a}(t)=\sum_{p} q_{p}(t)+\sum_{p \neq q} p_{p q}+\sum_{p \neq q} q_{p q}(t)
\end{gathered}
$$

In (9), each coefficient $P_{p}, Q_{p}, \Delta_{p q}, \Lambda_{p q}, \Delta_{q p}, \Lambda_{q p}$ of a corresponding multivector is at the same time a proportional rms value of a corresponding instantaneous power (13).

In fact, the equation (13) generates the keystone of the 
bridge between the magnitudes in frequency domain and their associated oscillations in time domain. Thus, the classic apparent power and non-active power values can also be interpreted in terms of Clifford coefficients and norms of corresponding oscillating components

$$
\begin{gathered}
S=\|\tilde{S}\|=\sqrt{\frac{2}{3} \sum_{p=q}\left\|p_{p}(t)\right\|^{2}+2 \sum_{p=q}\left\|q_{p}(t)\right\|^{2}+\sum_{p \neq q}\left\|p_{p q}(t)\right\|^{2}+\sum_{p \neq q}\left\|q_{p q}(t)\right\|^{2}} \\
\|\tilde{\Sigma}\|^{2}=\|\tilde{S}\|^{2}-\|\tilde{P}\|^{2}
\end{gathered}
$$

Note that the above mentioned power multivectors, $\tilde{S}$ and $\tilde{\Sigma}$ are the complements of the merely values $S=\|\tilde{S}\|$ and $\Sigma=\|\tilde{\Sigma}\|$. Both quantities (multivector and magnitude) lead to valid power equation representation, but it depends on the situation at hand to see which approach is more appropriate. Furthermore, in most cases direction and sense may not be required, but even in this case, the multivector can handle the problems equally or even better than the magnitude $\|\tilde{S}\|$. However, there are cases where direction and sense are necessary, whereby failing to distinguish the power components in terms of direction and sense may lead to erroneous results. Therefore, the Generalized Geometric Algebra [15] provides the necessary tools to extend the concepts of instantaneous and complex geometric analysis to higher dimensions.

\section{NUMERICAL EXAMPLE}

In this Section, a numerical example is proposed to illustrate the suggested non-active multivector concept and its natural capability to a one to one correspondence between its components and the terms of instantaneous non- active power. Units of physical quantities are those standards for the MKSA system and thus can be omitted.

Let voltage and current of a non-linear one-port circuit be

$$
\begin{gathered}
u(t)=\sqrt{2}[100 \sin t+100 \sin (3 t)] \\
i(t)=\sqrt{2}\left[50 \sqrt{2} \sin \left(t-45^{\circ}\right)-50 \sqrt{2} \sin (3 t)\right]
\end{gathered}
$$

and the corresponding geometric phasors are then

$$
\begin{gathered}
\tilde{U}=100 \sigma_{1}+50 \sigma_{3} \Rightarrow\|\tilde{U}\|=111.8 \\
\tilde{I}^{*}=70.71 e^{j 45} \sigma_{1}+20 e^{j 180} \sigma_{3} \Rightarrow\|\tilde{I}\|=73.48
\end{gathered}
$$

The instantaneous power equation and the apparent power multivector may be written

$$
\begin{aligned}
& \mathrm{s}(\mathrm{t})=\underbrace{\overbrace{5000}^{\mathrm{P}_{1}}\left[2 \sin ^{2}(\omega \mathrm{t})\right]}_{\mathrm{p}_{1}(\mathrm{t})}+\underbrace{\overbrace{(-1000)}^{\mathrm{P}_{3}}\left[2 \sin ^{2}(3 \omega \mathrm{t})\right]}_{\mathrm{p}_{3}(\mathrm{t})}+ \\
& \underbrace{-\overbrace{(5000)}^{\mathrm{Q}_{1}}[2 \sin (\omega \mathrm{t}) \cos (\omega \mathrm{t})]}_{\mathrm{q}_{1}(\mathrm{t})}+\underbrace{\overbrace{(-2000)}^{\Delta_{13}}[2 \sin (\omega \mathrm{t}) \sin (3 \omega \mathrm{t})]}_{\mathrm{P}_{13}(\mathrm{t})}+ \\
& +\underbrace{\overbrace{(2500)}^{\Delta_{31}}[2 \sin (3 \omega \mathrm{t}) \sin (\omega \mathrm{t})}_{\mathrm{p}_{31}(\mathrm{t})}] \underbrace{-\overbrace{(2500)}^{\Lambda_{31}}[2 \sin (3 \omega \mathrm{t}) \cos (\omega \mathrm{t})]}_{\mathrm{q}_{31}(\mathrm{t})}
\end{aligned}
$$

$$
\tilde{S}=[\underbrace{(5000-1000)}_{P=P_{1}+P_{3}}+j \underbrace{(5000+0)}_{Q=Q_{1}+Q_{3}}] \sigma_{0}+\underbrace{(4500+j 2500) \sigma_{31}}_{\bar{\Omega}_{31}^{\hat{n}}}
$$

and the conventional apparent power is given by

$$
S=\|\tilde{S}\|=8216
$$

The total instantaneous power is given by (22) and its waveform is depicted in Fig.2

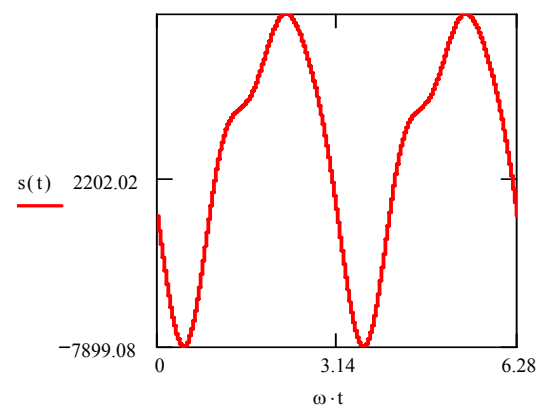

Fig.2. Total instantaneous power $\mathrm{s}(\mathrm{t})$

According to (14) and (15) the instantaneous active and non-active powers are given by

$$
p_{a}(t)=p_{1}(t)+p_{3}(t)
$$

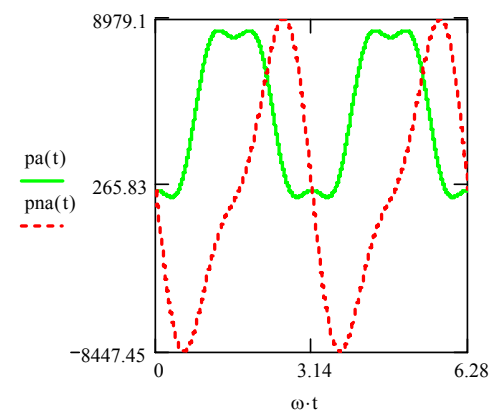

Fig.3. Active $(\mathrm{pa}(\mathrm{t}))$ and non-active $(\mathrm{pn}(\mathrm{t}))$ instantaneous powers

$$
p_{n a}(t)=q_{1}(t)+p_{13}(t)+p_{31}(t)+q_{31}(t)
$$

and these waveforms are shown in Fig.3.

Obviously, (22) and (23) can register the bidirectional active power flow occurring in this case $\left(P_{3}=-1000\right)$ and total active power is $5000-1000=4000$. This value coincides with the average power of (22).

Moreover, equation (22) also reveals a non active term

$$
\begin{aligned}
& \underbrace{\overbrace{(-2000)}^{\Delta_{13}}[2 \sin (\omega \mathrm{t}) \sin (3 \omega \mathrm{t})]}_{\mathrm{p}_{13}(\mathrm{t})}+\underbrace{\overbrace{(2500)}^{\Delta_{31}}[2 \sin (3 \omega \mathrm{t}) \sin (\omega \mathrm{t})]}_{\mathrm{p}_{31}(\mathrm{t})} \\
& =500[2 \sin (\omega \mathrm{t}) \sin (3 \omega \mathrm{t})]
\end{aligned}
$$

which amplitude value is $(-2000+2500)=500 \mathrm{VA}$. This is not the same value of that one deduced from (23), since $-2000 \sigma_{13}+2500 \sigma_{31}=4500 \sigma_{31}$. Although (22) and (23) are mathematically correct, the two terms that appeared in instantaneous power equation (22), $p_{13}(t)$ and $p_{31}(t)$, have the same nature despite their opposite signs. These possible erroneous results from the instantaneous power (22) occur due 
to the need of information concerning direction and sense of the components.

The non-active multivector terms (8) are always applicable to any power theory. Thus, in the Example, the $\operatorname{Im}\left\|\tilde{\Omega}^{\cdot}\right\|=5000$ value is equal to Budeanu and Slonim's reactive power. The magnitude $\|\tilde{\Sigma}\|=7176$ coincides with Fryze's reactive power and the result given by

$$
\sqrt{\left\|\operatorname{Im}\left\{\tilde{\Omega}^{\cdot}\right\}\right\|^{2}+\left\|\operatorname{Im}\left\{\tilde{\Omega}_{13}^{\wedge}\right\}\right\|^{2}}=5590
$$

is the same that the reactive power proposed by Shepherd [4], Sharon [5], and Czarnecki [9] on linear operation. The scattered power defined in [8] coincides with

$$
\left\|\operatorname{Re}\left\{\tilde{\Omega}_{13}^{\wedge}\right\}\right\|=4500
$$

and at the same time with the complementary reactive power of Sharon [5] and the active distortion of Slonim [6].

Thus, the proposed representation is unified and internally consistent with existing power equations. However, our equations are not derived from purely algebraic manipulations of the apparent power components in frequency domain, but rather they are a consequence of the power multivector. Today's accepted theories cannot explain the results obtained here.

\section{CONCLUSION}

In this paper, a new concept to the non-active power multivector under periodic $\mathrm{n}$-sinusoidal linear/non-linear operation has been presented. Moreover, power formulas have been given first in Clifford frequency domain and then in time domain. Further, a non-active power multivector has been developed, which condenses all power information and obeys the usual conservation law [16]. In this sense, it bridges the gap between frequency domain and time domain. The new non-active power multivector concept $\tilde{\Sigma}$ plays a similar role to the reactive power in the Steinmetz phasor model for the sinusoidal case; this multivector have a simple and compact expression and can identify the most relevant existing power equations.

Finally, from other point of view, the suggested representation can provide a new language for the design of compensator circuits, and optimization algorithms. The study of these applications is a task that deserves further research.

\section{LIST OF SYMBOLS}

$$
\begin{array}{ll}
\sigma_{1 . . k}= & \text { basis of Clifford algebra } \\
\odot & =\text { geometric product } \\
a \cdot b & =\text { inner product } \\
a \wedge b & =\text { outer product or bivector } \\
\left\|\tilde{Z}_{p}\right\|, Z_{p} & =\text { norm of p-th geometric-phasor } \\
(\tilde{Z})^{*} & =\text { conjugate element } \\
\tilde{U}_{p} & =\text { p-th voltage geometric-phasor }
\end{array}
$$

$\tilde{I}_{p} \quad=\mathrm{p}$-th current geometric- phasor

$\tilde{S} \quad=$ power multivector

$s(t) \quad=$ instantaneous power

$\tilde{\Sigma} \quad=$ non-active power multivector

$\tilde{\Omega}^{\cdot} \quad=$ complex-scalar

$\tilde{\Omega}^{\wedge} \quad=$ complex-bivector

$\varphi_{q} \quad=$ q-th impedance phase angle

$\Delta_{p q} \quad=$ pq-th $\{\operatorname{Re}\}$ part of distortion power multivector

$\Lambda_{p q} \quad=$ pq-th $\{\operatorname{Im}\}$ part of distortion power multivector

\section{REFERENCES}

[1] Budeanu CI. Puisances Reactives et Fictives. Instytut Romain de l'Energie 1927 Bucharest, Romania.

[2] Fryze S. Wik-,Blind, un Scheinleitung in Elektrischen Stromkreisen mit nichtsinusoidalem Verlauf von Strom und Spanung. Elekt. Z. 1932; 53: 596-599,625-627,700-702,

[3] Czarnecki LS. Budeanu and Frize:Two framework for interpreting power properties of circuits with nonsinusoidal voltages and currents. Electrical Engineering 1997;80: 359-367.

[4] Shepherd W and Zhakikhani P. Suggested definition of reactive power for nonsinusoidal systems. Proc. Inst.Elect. Eng. 1972; 119: 1361-1362.

[5] Sharon D. Reactive power definitions and power factor improvement in nonlinear systems. Proc Inst. Electr. Eng. 1973;120: 704-706.

[6] Slonim MA and Van Wyk JD. Powers components in a system with sinusoidal and nonsinusoidal voltages and/or currents. Proc. Inst. Elect Eng 1988; 135: 76-84.

[7] Willems JL and Ferrero A. Is there a Relationship between Non Active Currents and Fluctuations in the Transmitted Power?. ETEP 1998; 8: 265-270.

[8] Czarnecki LS.: Scattered and Reactive Current, Voltage and Power in Circuits with Nonsinusoidal Waveforms and Their Compensation. IEEE Trans. On Instrum. and Meas 1991; 40: 563-574.

[9] Czarnecki LS. Considerations on the reactive power in non-sinusoidal situations. IEE Trans. 1985; IM-34: 399-404.

[10] Czarnecki LS. Current's Physical components (CPC) Concept: A fundamental of Power Theory. IEE Trans. 1985; IM-34: 399-404.

[11] Emanuel AE. Powers in Nonsinusoidal Situations a Review of Definitions and Physical Meaning. IEEE Transactions on Power Delivery $1990 ; 5: 1377-1383$.

[12] Depenbrock M. The FBD- Method a Generally Aplicable Tool for Analyzing Power Relations. IEEE Transactions on Power Systems 1993; 8: 381-387.

[13] Sasdelli R. Montanari GC. Compensable Power for Electrical Systems in Nonsinusoidal Conditions. IEEE Trans. On Inst. and Meas 1994; 43: 592-598.

[14] Sommariva AM. Power Analysis of One-Ports Under Periodic MultiSinusoidal Operation, IEEE Trans. On Circuits and Systems.-I: Regular Papers 2006; 53: 2068-2074.

[15] Castilla M, Bravo JC, Ordoñez M, Montaño JC. Clifford Theory: A Geometrical Interpretation of Multivectorial Apparent Power. IEEE Transactions On Circuit and Systems I-Regular Papers 2008, 55: 33583367.

[16] Castilla M, Bravo JC, Ordoñez M. Geometric Algebra: A Multivectorial Prof. of Tellegen's Theorem in Multiterminal networks. IET Circuits, Devices and Systems 2008; 2, 383-390.

[17] Castilla M, Bravo JC, Ordoñez M, Montaño JC. The Geometric Algebra as a Power Theory Analysis Tool. Prezglad Elektrotechniczny 2009; 1 : 202-208.

[18] Bravo JC. Representación multivectorial de la Potencia Aparente en regímenes periódicos $\mathrm{n}$-senoidales aplicando Álgebras de Clifford. $\mathrm{Ph}$. D. Disertation. Univ. of Seville (Spain) 2008.

[19] Hestenes D, Sobczyk G. Clifford Algebra to Geometric Calculus: a unified language for Mathematics and Physics. Kluwer Academic 1986, Dordrech/Boston. 